\title{
Oxidación selectiva de glicerol empleando catalizadores de Pt y Pd soportados en zeolitas ZSM-11
}

\section{Selective oxidation of Glycerol using Pt and Pd Catalysts supported on ZSM-11 zeolites}

Presentación: 22/11/2019

Doctorando: Doctorado en Ingeniería- Mención Materiales

Nombre y Apellido: Eliana Diguilio

Centro de Investigación y Tecnología Química (CITeQ)- UTN- CONICET

ediguilio@frc.utn.edu.ar

Director/es:

Nombre y Apellido: Dra. Liliana Pierella

\section{Resumen}

Se sintetizaron zeolitas microporosas del tipo ZSM-11, con estructura MEL, por cristalización hidrotermal. Estos materiales fueron modificados con la incorporación de metales nobles como Au, Pty Pd, por impregnación en humedad incipiente a $80^{\circ} \mathrm{C}$. Luego, se agregó un promotor de Bismuto $(1 \% \mathrm{p} / \mathrm{p})$ por el método de co-impregnación. Por otro lado también, se incorporó $\mathrm{Au}(2 \% \mathrm{p} / \mathrm{p})$ por impregnación simultánea, a los catalizadores mono metálicos de Pt y Pd soportados, para evaluar el efecto combinado de estos metales. Después de esto, se realizaron tratamientos térmicos de desorción y calcinación para remover los restos de sales precursoras. Finalmente, todos los catalizadores fueron caracterizados por Difracción de Rayos X (DRX), área superficial BET, y mediante FTIR con adsorción de piridina para estudiar la fuerza acida de los mismos. Todos los materiales sintetizados y modificados fueron evaluados en la oxidación catalítica de glicerol (GLY). De las distintas alternativas existentes en la revalorización de GLY, nos hemos centrado en la oxidación selectiva en fase liquida, con el fin de obtener productos de mayor valor agregado. En este trabajo se evalúan las zeolitas ZSM-11 mono y bimetálicas en la oxidación selectiva de GLY empleando 02 puro como oxidante, obteniéndose valores de conversión del orden de 64\% mol, con un catalizador mono metálico de Pt-ZSM-11, y una selectividad a Ácido Láctico (LA) del 21\% mol. Este resultado mejoró empleando unos catalizadores bimetálicos de Au-Pt-ZSM-11 obteniéndose selectividades a LA del $45 \%$ mol.

Palabras claves: Glicerol, Au, Pt, Pd-ZSM-11, oxidación selectiva.

\begin{abstract}
Microporous zeolites of the type ZSM-11, with MEL structure, were synthesized by hydrothermal crystallization. These materials were modified with the incorporation of noble metals such as $\mathrm{Au}, \mathrm{Pt}$ and $\mathrm{Pd}$, by impregnation in incipient humidity at $80^{\circ} \mathrm{C}$. Then, a Bismuth promoter $(1 \% \mathrm{w} / \mathrm{w})$ was added by the co-impregnation method. On the other hand also, $\mathrm{Au}(2 \% \mathrm{w} / \mathrm{w})$ was incorporated by simultaneous impregnation, to the supported Pt and Pd mono metal catalysts, to evaluate the combined effect of these metals. After this, heat treatments of desorption and calcination were performed to remove the remains of precursor salts. Finally, all the catalysts were characterized by X-ray Diffraction (DRX), BET surface area, and by FTIR with pyridine adsorption to study their acid strength. All synthesized and modified materials were evaluated in the catalytic oxidation of glycerol (GLY). Of the different alternatives existing in the revaluation of GLY, we have focused on the selective oxidation in liquid phase, in order to obtain products with greater added value. In this work, the mono and bimetallic ZSM-11 zeolites are evaluated in the selective oxidation of GLY using pure 02 as oxidant, obtaining conversion values of the order of $64 \% \mathrm{~mol}$, with a mono-metallic catalyst of
\end{abstract}


Pt-ZSM-11, and a Lactic Acid (LA) selectivity of 21\% mol. This result was improved using bimetallic catalysts of AuPt-ZSM-11 obtaining selectivities at LA of $45 \% \mathrm{~mol}$.

Keywords: Glycerol, Au, Pt, Pd-ZSM-11, selective oxidation.

\section{Introducción}

En las últimas décadas, debido al aumento en el precio del barril de petróleo, ha surgido un gran interés en la producción de energía y combustibles a partir de fuentes renovables. Una de ellas resultó de gran interés en el área de la ciencia y tecnología: la producción de Biodiesel a partir de cultivos agropecuarios. Es bien conocido que la producción de Biodiesel genera un 10\% en peso de un subproducto llamado Glicerol (GLY), un alcohol con tres grupos hidroxilos (OH-). Esta molécula ha sido agrupada dentro de las "building blocks", a partir de la cual se pueden obtener productos de mayor valor agregado, de interés en la industria química, cosmética y farmacéutica, entre otras.

Se han planteado diferentes rutas de valorización de GLY, entre las que pueden mencionarse hidrogenólisis a 1,2 o 1,3 propanodiol (Barbelli M. L, et al., 2012), transesterificación a monoglicéridos y digliceridos (Corma, A, et al., 2005); deshidratación a acroleína (Akiyama, et al., 2009); halogenación a dicloropropanol y oxidación a aldeheídos, cetonas y ácidos (Gil. S, et al., 2011).

Para este fin se han desarrollado materiales catalíticos soportados basados en metales nobles como Pt, Pd, Au y de transición como Ni, Cr, Cu los cuáles brindan una enorme posibilidad en la obtención de compuestos oxigenados de interés. Existe una gran variedad de soportes estudiados, que van desde óxidos de metales de transición (Dai et al., 2018), carbón activado (Motta. D, et al., 2018) y zeolitas microporsas (Silva et al., 2017) hasta materiales mesoporosos (Tang. Z, et al., 2018). Por lo cual resulta interesante estudiar esta vía de valorización, en la cual el diseño de nuevos materiales se vuelve todo un desafío para lograr el control de la selectividad de la oxidación, obteniéndose así elevados rendimientos a productos de interés.

De las distintas alternativas existentes nos hemos centrado en la oxidación catalítica de GLY con el fin de obtener productos de mayor valor agregado, como Dihidroxiacetona (DHA), Gliceraldehido (GLA), Acido Glicólico (GLIC), Acido Glicérico (GA), Ácido Láctico (LA), habiéndose detectado otros productos de sobre oxidación como Ácido Acético (AA) y Fórmico (FA).

El ácido láctico (LA) es un compuesto de gran interés, usado ampliamente en la industria de alimentos, cosmética y farmacéutica. En particular, el ácido poliláctico tiene el potencial de reemplazar los plásticos de tereftalato de polietileno (PET) gracias a su biodegradabilidad y biocompatibilidad

Se estima que la demanda mundial de LA alcanzará los $600 \mathrm{kt}$ en 2020. Actualmente, LA se produce por fermentación de carbohidratos, proceso que requiere mucho tiempo e implica pasos de separación complejos. Debido a esto, la transformación química del GLY a LA está llamando la atención de la comunidad científica, y tecnológica (Dusselier, et al., 2013),(John, R. P. et al., 2007).

En el presente trabajo se exponen los resultados obtenidos en la oxidación selectiva de GLY, empleando 02 puro como oxidante, en condiciones leves, estudiando el efecto de los diferentes metales $\mathrm{Au}$, Pt y Pd, soportados sobre zeolitas microporosas de tipo ZSM-11.

\section{Experimental}

\section{Síntesis y modificación de materiales.}

Las Zeolitas microporosas del tipo ZSM-11 han sido sintetizadas en nuestro laboratorio, mediante tratamiento hidrotérmico a $140^{\circ} \mathrm{C}$ en un reactor autoclave recubierto con camisa de teflón, empleando como agente director de estructura el hidróxido de tetrapropilamonio (TPAOH) (Chu P. \& Corporation., 2009).

El gel de síntesis fue extraído del reactor, lavado con agua destilada hasta $\mathrm{pH}$ neutro y secado, luego, a $100{ }^{\circ} \mathrm{C}$ durante 24 h. Para eliminar el agente director de estructura, se somete al material a un tratamiento térmico de desorción en corriente de $\mathrm{N} 2$ desde temperatura ambiente hasta $500^{\circ} \mathrm{C}$. Posteriormente se procede a calcinar en aire $\left(500^{\circ} \mathrm{C}\right)$ durante $8 \mathrm{~h}$, para obtener el material en la forma Na-ZSM-11.

La forma NH4-ZSM-11 se obtiene mediante un intercambio iónico de la Na-zeolita con solución cloruro de amonio $(1 \mathrm{M})$ a $80^{\circ} \mathrm{C}$, durante $40 \mathrm{~h}$. Finalmente, para obtener la forma H-ZSM-11 se procedió a realizar nuevamente el tratamiento térmico (desorción - calcinación) antes mencionado a la zeolita en su forma NH4-ZSM-11.

El material sintetizado, en su forma NH4-ZSM-11, fue modificado con la incorporación de metales, como Pt y Pd (2\% $\mathrm{p} / \mathrm{p}$ ). El procedimiento fue llevado a cabo mediante impregnación en humedad incipiente a $80^{\circ} \mathrm{C}$, en un evaporador rotatorio, empleando Cloruro de Platino (IV) y Acetato de Paladio (II) como sales precursoras, respectivamente. Finalmente se llevaron a cabo los tratamientos térmicos de desorción en corriente de $\mathrm{N} 2$ y calcinación a 500을 $\mathrm{C}$ por $8 h$. 
También se procedió a incorporar un promotor de Bismuto (1\% p/p) a los catalizadores de Pt y Pd por coimpregnacion, en las mismas condiciones ya mencionadas.

Se ha reportado que el Bismuto sería un buen promotor para estos materiales, ya que orienta la reacción hacia la oxidación del grupo alcohólico secundario, facilitando así, la formación de DHA. Esto podría deberse a modificación de ciertas propiedades geométricas de las partículas, o también al bloqueo de ciertos sitios activos en el catalizador, obteniéndose así el producto deseado (Motta, et al., 2018).

A su vez, se prepararon catalizadores bimetálicos de Au-Pt/ZSM-11 y Au-Pd/ ZSM-11, por impregnación simultánea, con un $2 \% \mathrm{p} / \mathrm{p}$ de cada metal. Se empleó Acido cloro áurico como sal precursora y se siguió el mismo procedimiento descripto anteriormente.

\section{Caracterización de materiales.}

Las zeolitas ZSM-11 sintetizadas y modificadas, fueron caracterizadas por diferentes técnicas descriptas a continuación.

Difracción de rayos X (DRX) para corroborar estructura cristalina de los materiales, en un difractómetro Philips PW 3020 empleando radiación $\mathrm{K} \alpha$ de $\mathrm{Cu}$, de longitud de onda 0,15418 nm. Los datos de difracción se recogieron entre $2 \theta=$ 2-60을 a intervalos de $0,1^{\circ}$ y velocidad de $2^{\circ}$ por min.

Análisis de infrarrojo (FTIR) en un espectrómetro JASCO 5300, para determinar estructura, se prepararon pastillas al $0.05 \%$ de zeolita en $\mathrm{KBr}$, efectuando el análisis en la región de vibración de la red zeolítica (FTIR de fondo), entre 400-1800 cm-1. Con el fin de evaluar la fuerza acida de los materiales, (sitios ácidos de Lewis y Brönsted), se adsorbió piridina ( 3 Torr) a temperatura ambiente durante $12 \mathrm{~h}$, luego se desorbió durante una hora a diferentes temperaturas $\left(250,300\right.$ y $\left.400^{\circ} \mathrm{C}\right)$ a $10-4$ Torr. Los experimentos se llevaron a cabo utilizando una celda termostatizada con ventanas de CaF2 conectadas a una línea de vacío. La cantidad de sitios ácidos de Brönsted y Lewis se cuantificaron utilizando los datos de la literatura de los coeficientes de extinción molar integrados (Emeis C. A et al., 1993).

Determinación de Área Superficial por método BET llevada a cabo usando un equipo Micromeritics ASAP 2000 con absorción de $\mathrm{N} 2$ a $77 \mathrm{~K}$. Se evalúa el área expuesta de los catalizadores, antes y después de los tratamientos y modificaciones realizadas sobre estos.

Espectroscopía de Emisión Atómica con Plasma Inductivamente Acoplado (ICP) en un equipo Varian 715ES. Se determina la relación molar $\mathrm{Si} / \mathrm{Al}$ que conforman la estructura cristalina de las muestras, además del porcentaje de metal efectivamente incorporado en la zeolita.

\section{Experimentos catalíticos.}

Los materiales previamente sintetizados y modificados, fueron evaluados en la oxidación catalítica de glicerol (GLY) en fase liquida. El sistema de reacción consistió de un reactor de vidrio de tres bocas ( $50 \mathrm{ml}$ de capacidad) conectado a un condensador de reflujo, para evitar la posible evaporación de productos de reacción. El reactor se sumerge en un baño calefactor, para mantener la temperatura de reacción constante $\left(70^{\circ} \mathrm{C}\right)$ durante todo el experimento $(4 \mathrm{~h})$. Se deja circular oxígeno puro, con un flujo de $100 \mathrm{ml} / \mathrm{min}$ en el seno del líquido. Finalmente, se agrega una base como $\mathrm{NaOH}$, para generar el medio básico necesario, $\mathrm{NaOH} / \mathrm{GLY}=2$.

La identificación de productos de reacción se llevó a cabo por Cromatografía Líquida de Alta Presión (HPLC), en un equipo Jasco UV-975/ PU-980, empleando una columna Aminex HPX - 87H a 50 C, detector UV (210) e Índice de Refracción acoplados en serie. La fase móvil empleada fue solución de ácido sulfúrico ( $5 \mathrm{mM}$ ) con flujo de $0.6 \mathrm{ml} / \mathrm{min}$. La cuantificación de productos detectados se realizó mediante curvas de calibración empleando estándares puros.

\section{Resultados}

\section{Caracterización de los catalizadores.}

Los materiales sintetizados y modificados fueron caracterizados por diferentes técnicas. En la Tabla 1 se muestran los resultados de la caracterización fisicoquímica de los materiales empleados en este trabajo. El área superficial de los catalizadores es acorde a los valores que presentan las zeolitas del tipo ZSM-11.

Por XRD e IR (en zona de huella dactilar) se observó que la cristalinidad de los materiales no resultó afectada por la incorporación de metales, ni por los tratamientos térmicos posteriores efectuados sobre los mismos. Por ICP se determinó el contenido de metal incorporado efectivamente en la matriz, estos valores fueron acorde a los teóricos calculados.

Por análisis de infrarrojo con adsorción de piridina, se determinó la fuerza acida de estos materiales, como acidez de Lewis y de Bronsted, además de sitios ácidos totales $(\mathrm{B}+\mathrm{L})$. Los resultados obtenidos se resumen en la tabla 1 como relación de sitios ácidos de Bronsted/ Lewis (B/L). Cabe mencionar que el número de sitios ácidos totales de los catalizadores mono metálicos de Pt y Pd soportados sobre ZSM-11, aumentó después de la incorporación de Bi. Este efecto también se observa luego de la incorporación de Au a los catalizadores mono metálicos, debiéndose esto a un aumento mayoritario de los sitios de Lewis.

En la figura 1 se presentan los difractogramas correspondientes a la zeolita ZSM-11 modificadas con metales, donde se pueden observar las señales características de la matriz con estructura MEL $\left(2 \theta=7-8^{\circ}\right.$ y $2 \theta=21$ y $\left.22^{\circ}\right)$. Se presentan las señales características de los catalizadores mono metálicos (Pt, Pd / ZSM-11) y los mismos después de la 
incorporación de un promotor de Bi. Se presentan también, los catalizadores bimetálicos de Au-Pt y Au-Pd /ZSM-11. Pudo observarse la presencia de especies de los metales incorporados a la estructura (señales características de Au0, Pt0 y PdO) en las respectivas muestras.

Tabla 1. Propiedades fisicoquímicas de los catalizadores sintetizados.

\begin{tabular}{||l|l|l|l|c||}
\hline \multirow{2}{*}{ Catalizador } & $\begin{array}{l}\text { Metal } \\
(\% \mathrm{p} / \mathrm{p})\end{array}$ & $\begin{array}{l}\text { Área } \\
\text { superficial } \\
\left(\mathrm{m}^{2} / \mathrm{g}\right)\end{array}$ & $\begin{array}{l}\text { Cristalinidad } \\
\text { Relativa } \\
(\%)\end{array}$ & $\begin{array}{c}\text { Sitios } \\
\text { ácidos } \\
(\mathrm{B} / \mathrm{L})\end{array}$ \\
\cline { 2 - 5 } & ICP & BET & XRD & FTIR \\
\hline Pt-ZSM-11 & 1.8 & 383 & $>98$ & 4.84 \\
\hline Pd-ZSM-11 & 1.7 & 370 & $>97$ & 3.95 \\
\hline Pt-Bi-ZSM-11 & $1.7-0.88$ & 379 & $>97$ & 3.19 \\
\hline Pd-Bi-ZSM-11 & $1.7-0.91$ & 367 & $>98$ & 3.12 \\
\hline Au-Pt-ZSM-11 & $1.8-1.6$ & 378 & $>96$ & 4.12 \\
\hline Au-Pd-ZSM-11 & $1.7-1.7$ & 382 & $>96$ & 3.93 \\
\hline
\end{tabular}
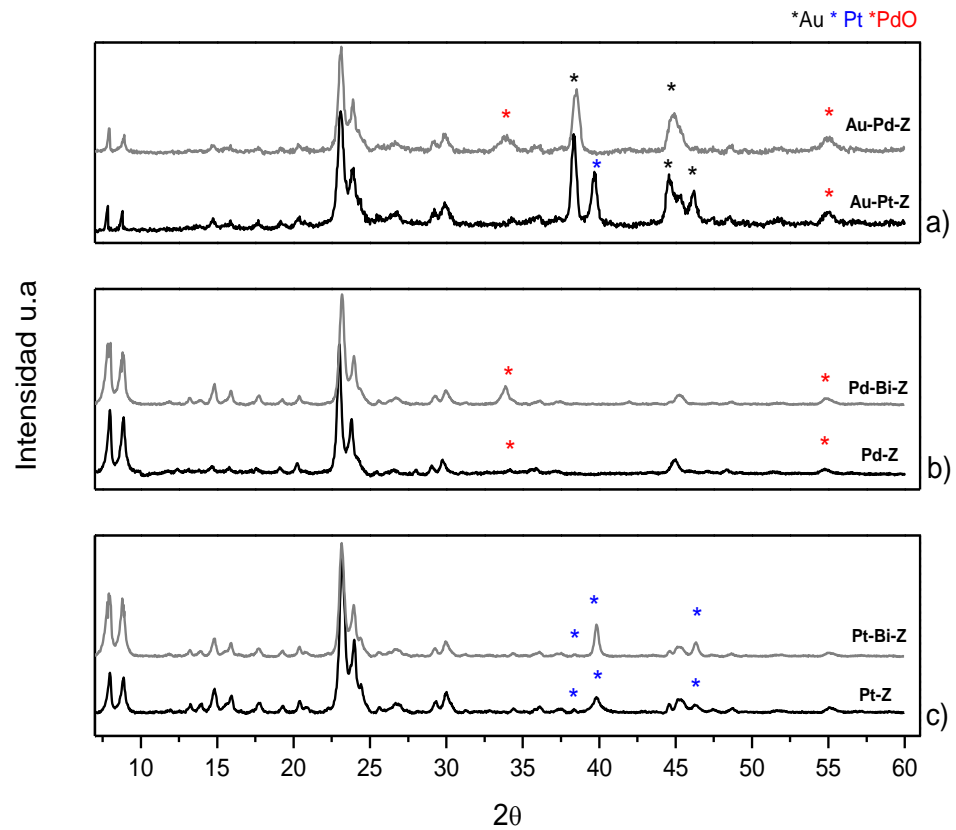

Figura 1. Difractograma de las zeolitas modificadas por incorporación de metales a) zeolitas bimetálicas Au-Pty AuPd/ ZSM-11, b) Pd y Pd-Bi/ ZSM-11, c) Pt y Pt-Bi/ ZSM-11.

\section{Actividad catalítica.}

Se evaluó la actividad catalítica de los materiales sintetizados y modificados con metales nobles como Au, y metales de transición como Pt y Pd, en la oxidación selectiva de GLY, en condiciones leves. En la tabla 2 se muestran los valores de conversión de GLY (\% mol) y selectividad (\% mol) a los distintos productos detectados en las siguientes condiciones: solución acuosa de GLY $0.25 \mathrm{M}$, a temperatura de $70^{\circ} \mathrm{C}, 100 \mathrm{ml} / \mathrm{min}$ de 02 , relación molar $\mathrm{NaOH} / \mathrm{GLY}=$ 2, durante 4 horas).

En primer lugar, cabe destacar, que los resultados obtenidos con catalizadores monometálicos de Pt-ZSM-11 y PdZSM-11 sin la presencia de una base en el medio de reacción, arrojaron valores muy bajos de conversión de GLY entre 10 y $17 \%$ mol (no presentados en tabla 3). Este comportamiento puede atribuirse a que el oxidante (02 puro) necesita de los $\mathrm{OH}$ - aportados por la base, para producir la deshidrogenación. Este es el primer paso en la reacción de oxidación pudiendo generarse DHA y/o GLA, productos de oxidación primaria. Debido a esto, numerosos estudios han destacado el rol indirecto del 02 en reacciones de oxidación de alcoholes. Durante la activación del 02 comienza a producirse H2O2 en el medio de reacción, favoreciendo el rompimiento de enlaces C-C, obteniéndose así, ácidos orgánicos (Brushan N. Zope et al. 2010) 
La conversión de GLY, empleando Pt-ZSM-11, fue del 64\% mol, en las condiciones ya mencionadas detectándose los siguientes productos de reacción: GLA, GA, LA y AA, entre otros. El LA es uno de los productos de mayor interés, obtenido aquí, directamente desde el GLY y en condiciones suaves de reacción, siendo muy interesante y prometedor este resultado. Cabe mencionar aquí, que este material es el que mayor relación de sitios ácidos presenta $(\mathrm{B} / \mathrm{L}=4.84)$.

Por otro lado, es bien sabido que el Bi puede actuar como un promotor en estos catalizadores, bloqueando ciertos sitios activos del metal, facilitando así, la formación de productos de oxidación primaria como GLA. Mencionamos aquí, que no se ha detectado DHA entre los productos de reacción, posiblemente debido a su dificultad para desorberse desde los sitios activos hacia la superficie del catalizador, y continuaría reaccionando a los productos detectados.

Con la incorporación de Bi al catalizador de Pt-ZSM-11 se mantuvieron valores de conversión de GLY en 60 \% mol y la selectividad a productos también fue similar. Sin embargo, resulta interesante destacar que, al incorporar Bi, no se obtuvo AA, un compuesto de sobreoxidación del LA. Esto podría deberse a un efecto atenuante del Bi sobre el poder oxidante del Pt (Motta et al., 2018).

Los catalizadores de Pd-ZSM-11 y Pd-Bi-ZSM-11 resultaron en conversiones menores de GLY (38 \% y $20 \%$ mol respectivamente), con la obtención de GA como producto mayoritario ( 74 y $95 \% \mathrm{~mol}$, respectivamente). Este producto resulta de interés para la industria cosmética (Johnston, et al., 2002).

Evaluando los catalizadores bimetálicos, Au-Pt-ZSM-11 y Au-Pd-ZSM-11, se obtuvieron mejores resultados con respecto a los catalizadores mono metálicos de Pt y Pd. Si bien los valores de conversión de GLY fueron menores, que sus respectivos monometálicos, se produjo un incremento considerable en la selectividad a LA, que resultó en 44.57 $\%$ mol para el Au-Pt-ZSM-11, y $26.90 \%$ mol para el Au-Pd-ZSM-11, con muy baja selectividad a AA ( 1\%), en ambos casos. Estos resultados podrían deberse a un efecto sinérgico entre estos metales Au-Pt y Au-Pd y a la relación de sitios ácidos B/L (ver Tabla 1). Han sido reportados diversos estudios empleando catalizadores de Au soportados, que resultaron muy específicos, estables frente a una posible lixiviación y resistentes a la sobre oxidación por 02 (Mallat \& Baiker, 2004) (Prati \& Rossi., 1998), confirmando los resultados obtenidos.

Tabla 2. Actividad catalítica de los materiales en la oxidación de glicerol

\begin{tabular}{||c|c|c|c|c|c|c||}
\hline \hline Catalizador & GLY & GLA & GLIC & GA & LA & AA \\
\hline Pt-ZSM-11 & 63.48 & 23.67 & - & 33.98 & 21.11 & 7.74 \\
\hline Pt-Bi-ZSM-11 & 59.98 & 17.94 & - & 29.96 & 14.13 & - \\
\hline Pd-ZSM-11 & 38.02 & 1.18 & 0.83 & 74.00 & - & - \\
\hline Pd-Bi-ZSM-11 & 20.91 & 2.63 & - & 95.07 & - & - \\
\hline Au-Pt-ZSM-11 & 32.15 & 21.45 & - & 30.13 & 44.57 & 1.17 \\
\hline Au-Pd-ZSM-11 & 25.13 & 21.55 & - & 31.24 & 26.90 & 0.42 \\
\hline \hline
\end{tabular}

\section{Conclusiones.}

Se han sintetizado catalizadores del tipo zeolíticos, con estructura MEL, con posterior incorporación de metales nobles como Au, Pt y Pd, en un 2\% p/p, con elevados porcentajes de cristalinidad y pureza. Estos materiales fueron evaluados en la oxidación selectiva de GLY en medio básico ( $\mathrm{NaOH})$, empleando 02 puro como oxidante, a $70^{\circ} \mathrm{C}$ y durante 4 h de reacción.

Evaluando los resultados obtenidos, podemos decir que el catalizador monometálico de Pt-ZSM-11 generó la mayor conversión de GLY ( $\sim 64 \% \mathrm{~mol})$ después de $4 \mathrm{~h}$ de reacción. Los productos de mayor interés detectados, fueron LA y GA (21.11 y $33.98 \%$ mol, respectivamente). Estos resultados mejoraron al emplear un catalizador bimetálico de AuPt-ZSM-11, con selectividades cercanas al 45\% mol de LA.

A su vez, empleando un catalizador de Pd- ZSM-11, este resultó muy selectivo a GA, obteniéndose un 74\% mol. Luego de la incorporación de un promotor, se logró mejorar la selectividad, alcanzando un 95\% mol de GA con Pd-BiZSM-11, demostrando así, el efecto del Bi.

\section{Referencias}

Akiyam, M., Sato, S., Takahashi, R., Inui, K., \& Yokota, M. (2009). Applied Catalysis A : General Dehydration hydrogenation of glycerol into 1,2-propanediol at ambient hydrogen pressure, 371, 60-66.

https://doi.org/10.1016/j.apcata.2009.09.029 
Barbelli, M. L., Santori, G. F., \& Nichio, N. N. (2012). Bioresource Technology Aqueous phase hydrogenolysis of glycerol to bio-propylene glycol over Pt - Sn catalysts. Bioresource Technology, 111, 500-503.

https://doi.org/10.1016/j.biortech.2012.02.053

Chu, P., \& Corporation, M. O. (2009). United States Patent Office. Journal of the American Society for Naval Engineers, 39(4), 620-622.

Corma, A., Bee, S., Hamid, A., Iborra, S., \& Velty, A. (2005). Lewis and Brönsted basic active sites on solid catalysts and their role in the synthesis of monoglycerides, Journal of Catalysis 234 (2005) 340-347. https://doi.org/10.1016/j.jcat.2005.06.023

Dai, J., Xiao, X., Duan, S., Liu, J., He, J., Lei, J., \& Wang, L. (2018). Synthesis of novel microporous nanocomposites of ZIF-8 on multiwalled carbon nanotubes for adsorptive removing benzoicacid from water. Chemical Engineering Journal, 331(August 2017), 64-74. https://doi.org/10.1016/j.cej.2017.08.090

Dusselier, M., Van Wouwe, P., Dewaele, A., Makshina, E., \& Sels, B. F. (2013). Lactic acid as a platform chemical in the biobased economy: The role of chemocatalysis. Energy and Environmental Science, 6(6), 1415-1442. https:7/doi.org/10.1039/C3EE00069A

Emeis, C. A, (1993). Determination of Integrated Molar Extinction Coefficients for Infrared Absorption Bands of Pyridine Adsorbed on Solid Acid Catalysts. Journal of Catalysis 141, 347-354.

https://doi.org/10.1006/jcat.1993.1145

Gil, S., Marchena, M., Sánchez-silva, L., Romero, A., \& Sánchez, P. (2011). Effect of the operation conditions on the selective oxidation of glycerol with catalysts based on Au supported on carbonaceous materials. Chemical Engineering Journal, 178, 423-435. https://doi.org/10.1016/j.cej.2011.10.048

John, R. P., Nampoothiri, K. M., \& Pandey, A. (2007). Fermentative production of lactic acid from biomass: An overview on process developments and future perspectives. Applied Microbiology and Biotechnology, 74(3), 524-534. https://doi.org/10.1007/s00253-006-0779-6

Johnston, P., Griffin, K., McMorn, P., Hutchings, G. J., \& Carrettin, S. (2002). Selective oxidation of glycerol to glyceric acid using a gold catalyst in aqueous sodium hydroxide. Chemical Communications, (7), 696-697. https://doi.org/10.1039/B201112N

Mallat, T., \& Baiker, A. (2004). Oxidation of Alcohols with Molecular Oxygen on Solid Catalysts. Chemical Reviews, 104(6), 3037-3058. https://doi.org/10.1021/cr0200116

Motta, D., Sanchez, F. J., Dimitratos, N., Villa, A., \& Prati, L. (2018). An investigation on AuPt and AuPt-Bi on granular carbon as catalysts for the oxidation of glycerol under continuous fl ow conditions. Catalysis Today, 308, 50-57. https://doi.org/10.1016/j.cattod.2017.10.012

Prati, L., \& Rossi, M. (1998). Gold on carbon as a new catalyst for selective liquid phase oxidation of diols. Journal of Catalysis, 176(2), 552-560. https://doi.org/10.1006/jcat.1998.2078

Silva, T. Q., Maurício, B., Santiago, A. A. C., Santana, D. O., Cruz, F. T., Andrade, H. M. C., \& Mascarenhas, A. J. S. (2017). Gas phase glycerol oxidative dehydration over bifunctional V / H-zeolite catalysts with different zeolite topologies. Catalysis Today, 289, 38-46. https://doi.org/10.1016/j.cattod.2016.08.011

Tang, Z., Fiorilli, S., Heeres, H. J., Pescarmona, P. P., \& Accepted, J. (2018). Multifunctional heterogeneous catalysts for the selective conversion of glycerol into methyl lactate.

https://doi.org/10.1021/acssuschemeng.8b02277 